\title{
Reviews in Philosophy
}

\author{
Christoph Lumer ${ }^{1}$
}

Accepted: 7 April 2018 / Published online: 19 April 2018

(C) Springer Science+Business Media B.V., part of Springer Nature 2018

Recent years have borne witness to a mainstreaming within scientific publications that is unprecedented in history since the emergence of scientific journals. This is especially true of philosophical journals and even more so of ethics journals. Here I will address one mechanism contributing to this development, namely the review process that attends decision-making in the publication of journal articles.

Reviews, of course, have an important filtering function in the exclusion of poor articles. But they also relentlessly screen out much of what would be more than worth publishing, I'm afraid just many of the most innovative and original contributions. And they let pass a lot of conventional papers that lack substantive value. It is this kind of review that I would like to discuss.

Good reviews, serving the cause, recognize the actual value of an article, provide indications of errors, and moreover propose useful suggestions for improvement. Of course, such reviews do exist; but they are too rare. Some types of bad reviews, leading to the rejection of good articles, originate in the following ways:

The nitpicker and formalist criticizes formal trifles - rightly or wrongly: Here a quotation is missing, the page number; a premise is not justified (although it would not be difficult to justify it). ...

The overconfident reviewer believes that he knows what is en vogue in the present discussion or what must be discussed in any case (although this relevance originates more from his own idiosyncrasies) and demands respective discussions from the author.

The devotee of the canon wants a discussion of a certain set of theories, theses, or debates, because this is - allegedly - the state of science. Whether this discussion is actually relevant to the topic of the article, or would undermine its theses, does not interest him.

The abusive philosophical opponent sees in the article a threat to the reception of his own theory, for which he wants to achieve in any case a form of publication sovereignty. The appearance of articles coming from other directions and which do not (nevertheless) appreciate the reviewer's theory or even dare to criticize his position must be blocked at all costs - the

Christoph Lumer

lumer@unisi.it

1 University of Siena, Siena, Italy 
better the criticism, the more important it is to prevent its publication. Pretexts for this are easily to be found.

All this leads to the rejection of good articles or to requests for changes that do not really improve the article, on the contrary often make it worse.

Some general mechanisms involved in such false negatives are the following: the thesis or theory developed in the article is - surprisingly - not considered at all and not even mentioned in the review; probably the reviewer did not understand it. Or it is acknowledged, but not duly evaluated in relation to existing theories. Considering the theory or thesis actually developed in the paper, the review is preoccupied with trivialities concerning mainly formalities. Instead of concentrating on the topic in hand, demands for supplementation or restructuring are made regardless of the context and space available - while at the same time criticizing that the article is too long anyway.

Some types of bad reviews, on the other hand, which lead to acceptance of very mediocre, superfluous or poor articles include:

The quick reviewer has looked at the article only briefly and uses the criterion: Does the article meet certain minimum standards, and does it propound anything new? The value of this innovation is not questioned.

The insecure cannot make any real sense of the article, probably it is outside of his area of competence. To conceal this, he accepts the article, in order to be on the safe side.

The partial referee lets articles of his own school or to which he is ideologically sympathetic pass, which, however, should actually be rejected.

In the foregoing ways, then, articles of dubious value pass: critiques and meta-critiques of special objections (raised particularly within the same journal) against certain theories (often nobody would probably have registered or taken this objection seriously anyway); discussions of minutiae within a real or current "classic"; defences of theses that completely ignore the state of knowledge in other areas; "clever" differentiations that, however, do not help anyone.

As a result, the published articles are conventional. They routinely consider the same theoretical approaches, which once gained a certain predominance, or deal with fashionable topics that are declinated in all ramifications; new approaches instead are censored. The clever and quick producer takes up a current discussion topic, picks up on the most important current contributions to it, and produces a compromise proposal, a "tricky" (but unsustainable) way out of a conflict, a variant of already presented solution proposals. These ad hoc producers are well-informed about the current discussion, but lack real basic, substantive knowledge and any sense of the real problem. - Writing styles from non-English cultures disappear. Papers that are difficult to read and have minimal positive content are produced: only after a lengthy discussion of alternative approaches and various predecessor versions of the author's own proposal is the actual thesis presented, typically at the end of the paper - by which point, unfortunately, there is no room for its argumentative defence, or the author, quite generally, refers to the previous discussion, in which (allegedly) everything has already been proved.

Why, then, are many reviews so bad? Reviewers often lack professional and human sovereignty. Rofessional sovereignty would grow out of experience with the whole spectrum of views in one's own field; sovereignty would require broad knowledge also in neighbouring philosophical disciplines; sovereignty would presuppose a certain knowledge of the empirical disciplines to which reference should be made; but professional sovereignty would also be based on a broad methodological and argumentative training, which, then, leads not only to insisting on the observance of the relevant methodological and argumentative criteria but also to a methodological openness towards deviating approaches or new ideas, which cannot yet be 
fully elaborated or require a more extensive explanation. The reviewer who lacks professional sovereignty instead adheres to prevailing standards, and latest fashions, which she then supports and encourages because she does not (yet) have a sense for originality, the unusual and quality. Moreover, in many cases there is a lack of human sovereignty and modesty, which would consist in understanding one's own limitations, tolerance towards approaches deviating from one's own opinion, and acknowledgement and recognition of innovations in other approaches. Therefore, reviewers who lack sovereignty cling to formalities and their own narrow area of knowledge. The widespread lack of sovereignty is in turn largely due to the fact that in many cases only junior scholars can be recruited for the ungrateful task of peer review, who, however, are still green behind the ears. - Another, rather trivial reason for the low quality of many reviews is that the respective reviewers devote far too little time to their task and make a hasty assessment, inter alia, because of the ingratitude of the task and the abundance of the requested reviews. A further reason is also the lack of suitable standards for making assessments concerning primarily substance and content. Moreover, the quality of expert reports is insufficiently checked by supervisory instances.

How, then, can the quality of reviews be improved while avoiding the risk of exacerbating the (already pandemic) bureaucratized and incredibly time-consuming review process? (The abundance of referee reports and the high costs of a good individual report reduce the chances of getting good reviewers and reports.) Ethical Theory and Moral Practice could provide reviewers with guidelines for good reviews that encourage them to consider the really important matters. (The "Guidelines for Reviewers" of Informal Logic, e.g., provide some helpful suggestions.) If an article is rejected, the author could be given an opportunity for comment. Editors could monitor the reports and, if necessary, correct or relativize them. 